\title{
Safety Strategies for Women Experiencing Intimate Partner Violence During the COVID-19 Pandemic: a Scoping Review Protocol
}

\section{ZoeTipa ( $\sim$ zoe.tipa@aut.ac.nz )}

Auckland University of Technology https://orcid.org/0000-0003-4366-9997

\section{Te Wai Barbarich-Unasa}

Auckland University of Technology

\section{Shyamala Nada-Raja}

University of Otago

Jane Koziol-McLain

Auckland University of Technology

\section{Protocol}

Keywords: Intimate Partner Violence, Safety Strategies, COVID-19, Women, Family Violence, Domestic Violence, Domestic Abuse, Safe, Help, Whānau Violence, Safety Plans, Support Plans, Māori, Wahine, Battered Women, New Zealand, Pandemic, Prevention, Abuse, Coronavirus, Gender-based violence, Reproductive health, SARS-CoV-2 virus, Indigenous

Posted Date: July 1st, 2021

DOI: https://doi.org/10.21203/rs.3.rs-654912/v1

License: (9) This work is licensed under a Creative Commons Attribution 4.0 International License. Read Full License 


\section{Abstract}

\section{Introduction}

The health and social impacts of the COVID-19 pandemic evident internationally are mirrored in New Zealand. ${ }^{1}$ With reports of escalating Intimate Partner Violence (IPV), there is a need for services supporting women and families to adapt to the changing COVID-19 environment. An interactive, individualised web-based safety-decision aid called isafe is being redeveloped to reflect these changes across Aotearoa New Zealand. Due to the social distancing requirements and fluidity of COVID-19 alert levels, safety strategies supporting women experiencing IPV need to align with the latest evidence within the context of COVID-19. This scoping review is designed to identify the range of extant safety strategies for women experiencing IPV suggested as effective during the COVID-19 pandemic.

\section{Methodology and method}

We will follow the scoping review methodology framework proposed by Johanna Briggs Institute (JBI), ${ }^{2}$ originally developed by Arksey and $0^{\prime}$ Malley ${ }^{3}$ and further refined by Levac ${ }^{4}$. The search strategy includes all study designs, and editorials and grey literature. Two reviewers will screen each article title and abstract against inclusion and exclusion criteria before independently screening the full text of eligible articles. Grey literature will be searched using existing websites and organisations that provide links to resources focussed on family violence (Table 1). Culturally informed strategies supporting Māori women and their whānau (family) will be specifically identified within the full text screening process. Data will be analysed using qualitative narrative synthesis to consider the strategies and their application to the New Zealand context. This will inform the development of isafe to support women experiencing violence during COVID-19 and beyond.

\section{Ethics and dissemination}

Ethical approval was not required for this study. Results will be used to inform a future study in which key stakeholder groups will evaluate the usability of isafe in preparation for deployment. Findings of this review will be published in journals and validated with stakeholders connected with the isafe project.

\section{Introduction/background}

Intimate Partner Violence (IPV) is recognised as a worldwide public health issue associated with increased morbidity and mortality, with significant health and social consequences of affected individuals ${ }^{5}$. IPV can include physical violence, sexual violence, psychological abuse and controlling behaviours by a current or past intimate partner. It is also one of the most common causes of harm to women worldwide ${ }^{6}$. Increased physical health problems such as injury, chronic pain, gastrointestinal, and gynaecological signs, as well as mental health issues such as depression and post-traumatic stress disorder are all identified as issues arising from IPV ${ }^{7}$. There is an ongoing need for resources and tools that women can access in ways it is safe for them to do so. Evidence-based safety strategies support 
women to protect themselves from both experiencing IPV and from the ongoing effects of IPV ${ }^{8}$. Therefore, the aim of this scoping review is to identify and describe the safety strategies identified in existing literature, to inform the development of a web-based safety decision tool for women experiencing IPV during COVID-19.

IPV has been described by the Executive Director of the United Nations Women as a "shadow pandemic" during COVID-19 ${ }^{1}$. Government authorities and non-government partners worldwide are reporting escalating IPV as countries grapple with the impacts of COVID-19. The coronavirus pandemic has influenced the lives of all people in New Zealand, with those vulnerable to family harm particularly impacted. Family violence is estimated to cost the New Zealand economy $\$ 4.1$ billion to $\$ 7.0$ billion dollars annually ${ }^{9}$. Before the coronavirus pandemic, 1 in 3 women in New Zealand experienced physical and/or sexual violence from an abusive partner in intimate relationships in their lifetime ${ }^{10}$. However, the current pandemic has amplified cases of IPV against women, with up to three times as many IPV cases compared to the same time last year ${ }^{1}$. This experience is echoed in New South Wales with $45 \%$ of IPV frontline workers reporting an escalation of IPV cases and $71 \%$ reporting increased complexity related to COVID-1911.

Māori are overrepresented in family violence statistics with 1 in 2 Māori women experiencing IPV in their lifetime compared with 1 in 3 non-Māori women ${ }^{10}$. Māori women are also three times as likely to be victims of homicide than non-Māori ${ }^{12}$. For Indigenous women, stigma and structural racism are common barriers to disclosing their experience of abuse to a formal service ${ }^{13}$. The social and structural entrapment experienced by women in abusive relationships, particularly for Māori women, is compounded by the impact of measures needed to contain COVID-19 ${ }^{14}$. Therefore, any initiatives designed to support women experiencing IPV need to be responsive to the social and cultural needs of Māori.

The ongoing COVID-19 crisis brings emotional stress, economic strain, disruption of roles and responsibilities combined with restricted movement and social isolation. The stay-at-home policies have effectively reduced the spread of the disease leading to worldwide implementation to varying degrees ${ }^{15}$. IPV intensifies while women and their children are isolated from the people and resources that could help them ${ }^{16}$. Home is not a safe place for everyone, especially for those who may have to work from home or self-isolate at home with a partner who is agitated due to the social distancing restrictions during a pandemic. IPV victims during COVID-19 need alternative ways to stay safe as the usual channels of support may be compromised by the requirements surrounding social distancing ${ }^{1}$. Internationally there are reports of escalating IPV and a need for service innovations to support the types of help needed by victims of IPV at this time ${ }^{17}$.

In Aotearoa/New Zealand, an interactive, individualised web-based safety-decision aid (isafe) was developed in 2014 and tested in a randomised controlled trial. The isafe tool includes risk assessment and priority setting activities for safety decision making and the development of a personalised safety 
action plan for women experiencing IPV. isafe is currently being retooled for the COVID-19 context and will be deployed across Aotearoa/New Zealand. This review will identify current and emerging best practice related to safety strategies for women experiencing IPV during the COVID-19 pandemic to inform the safety plans within isafe. Dissemination of isafe nationwide has the potential to contribute to reducing IPV severity and improve health and wellbeing during the pandemic alerts, particularly for Māori women who have access to technology and the internet. isafe offers an alternative to formal help seeking and is potentially an economically sustainable resource. With effective community deployment, the tool may be a link for women to seek help from formal services, or for family advocates to incorporate within their assessment processes.

A scoping review was identified as the most appropriate method to identify extant COVID-19 related safety strategies within the literature. Scoping reviews are an increasingly popular method to review a broad range of health research evidence ${ }^{4}$. As a method to support knowledge synthesis, scoping reviews have been used to answer a range of health-related research questions and have potential to advance health care practice, policy and research ${ }^{18}$. In undertaking scoping reviews, researchers can critically examine the extent, range, and nature of the research activity in order to summarise and disseminate research findings and/or identify gaps in the existing literature ${ }^{4}$.

A preliminary search of MEDLINE, the Cochrane Database of Systematic Reviews and JBI Evidence Synthesis was conducted and no current scoping reviews on the topic were identified. Initial search of databases and google identified multiple resources that may fulfil the inclusion criteria. The objective of this scoping review is to identify the safety strategies in the literature that are specific to the COVID-19 context. An analysis of these safety strategies alongside discussions with community advocates about the application within Aotearoa New Zealand will inform the development of isafe to support women experiencing violence during COVID-19 and beyond.

\section{Review Question}

Our scoping review seeks to answer, what safety strategies exist for women experiencing intimate partner violence during the COVID-19 pandemic?

The scoping review is designed to meet the following objectives:

- Identify safety strategies designed to support women experiencing intimate partner violence during the COVID-19 pandemic.

- Analyse appropriateness of strategies from policy, agency and service user perspectives.

- Identify culturally informed safety strategies designed to support Indigenous Māori women.

\section{Methodology And Method}

\section{Inclusion Criteria}




\section{Type of participant}

Research involving women and women with families who are exposed to IPV will be included within this scoping review. Research focusing on men and children will be excluded from the scoping review. Articles that support Māori women and whānau and Indigenous populations will be identified separately within the data extraction tool (Table 2).

\section{Concept}

The key concept of interest is the identification of safety strategies for women experiencing IPV. Within the literature, a variety of different terms such as safety plans, support plans, domestic violence, and family violence have been used interchangeably to articulate how to support women and their families who are exposed to IPV. The types of safety strategies and the development of and response to safety strategies will be extracted using the data extraction template (Table 2). This is to consider whether strategies have been informed and evaluated by women they are designed to support or whether they are led by organisations and/or policy.

\section{Context}

The context for this scoping review is the COVID-19 pandemic, and more specifically, the identification of safety strategies during the COVID-19 pandemic. Safety strategies identified in literature outside the COVID-19 pandemic will be excluded from this scoping review. Safety strategies for women internationally will be included within the review. There will also be an emphasis on searching for grey literature within a New Zealand context to identify strategies that apply to Māori women.

\section{Type of sources}

This scoping review will consider all qualitative, quantitative, experimental, and non-experimental studies including randomised controlled trials and non-randomised controlled trials. Reviews and systematic reviews of programs that meet the inclusion criteria will also be retrieved. Grey literature will also be considered for inclusion in this scoping review. Grey literature will be searched using existing websites and organisations that provide links to resources focussed on family violence. Studies published in English and published from 2019 onward will be included within the review.

\section{Methods}

There is currently no scoping review protocol registered that is focussed on identifying safety strategies for women experiencing IPV during COVID-19. This scoping review will be conducted in accordance with the Johanna Briggs Institute (JBI) methodological framework proposed by Peters, et al. ${ }^{2}$ originally developed by Arksey and $0^{\prime}$ Malley ${ }^{3}$ and further refined by Levac ${ }^{4}$. We will follow the nine-stage process outlined by Peters et al. ${ }^{2}$ which includes:

1) Defining and aligning the objective/s and question/s

2) Developing and aligning the inclusion criteria with the objective/s and question/s 
3) Describing the planned approach to evidence searching, selection, data extraction, and presentation of the evidence

4) Searching for the evidence

5) Selecting the evidence

6) Extracting the evidence

7) Analysis of the evidence

8) Presentation of the results

9) Summarizing the evidence in relation to the purpose of the review, making conclusions and noting any implications of the findings

Two reviewers will use the inclusion and exclusion criteria to screen titles and abstracts using a systematic reviews production tool (Covidence ${ }^{19}$ ) for inclusion in the full review. Full texts of eligible articles will be screened by two independent reviewers and evidence will be extracted using the criteria outlined in the data extraction template (Table 2). Given the disproportionate rates of family violence for Māori, strategies specifically designed to support Māori women and their whānau (family) will be identified separately within the data extraction process.

\section{Search Strategy}

The search strategy will aim to locate both published and unpublished studies. An initial search of MEDLINE was undertaken to identify articles on the topic. Terms related to IPV including domestic violence, battered women, or partner abuse. Terms related to safety strategies including help seeking, helping behaviour, or seeking to end violence were also used. The initial search terms included: Intimate Partner Violence, Safety Strategies, COVID-19, Women and Indigenous. The text words contained in the titles and abstracts of relevant articles, and the index terms used to describe the articles were used to develop a full search strategy (Table 1). The search strategy, including all identified key words and index terms, will be adapted for each included database and/or information source. The reference lists of all included sources of evidence will be screened for additional studies. 
Table 1

Abstract and Title Database search strategy

\section{Search terms}

(1) Keywords for "intimate partner violence":

("IPV" OR "family violence" OR "domestic violence" OR "whānau violence" OR "battered women" OR "domestic abuse" OR "gender-based violence" OR "abuse") AND

(2) Keywords for "COVID-19":

("COVID-19 pandemic" OR "pandemic" OR "coronavirus" OR "SARS-CoV-2 virus") AND

(3) Keywords for "safety strategy":

("safety plan" OR "help seeking" OR "plan" OR "response" OR "support" OR "safe" OR "help" OR "support plans") AND

(4) Keywords for "women":

("girls" OR "female" OR "wahine" OR "kōtiro" OR "gender" OR reproductive health) AND

(5) Keywords for "Indigenous"

("Māori” OR "kaupapa Māori” OR "Māori-led" OR "first nations" OR "first peoples"

\section{Databases}

CINAHL (via Ovid), MEDLINE (via Ovid), Cochrane Library (via Wiley), Scopus (via EBSCO), SocINDEX (via EBSCO), PsychINFO, New Zealand Family Violence Clearinghouse, and Indigenous open access journals - MAI and Te Kaharoa

\section{Study Source of Evidence Selection}

Following the search, all identified citations will be collated and uploaded into EndNote and duplicates removed. Then uploaded into Covidence, ${ }^{19}$ the full text, titles and abstracts of selected citations will be assessed in detail against the inclusion criteria by two or more independent reviewers. Conflicts will be resolved through discussion with research team members. Reasons for exclusion of sources of evidence at full text that do not meet the inclusion criteria will be recorded and reported in the scoping review. The results of the search and the study inclusion process will be reported in full in the final scoping review and presented in a Preferred Reporting Items for Systematic Reviews and Meta-analyses extension for scoping review (PRISMA-ScR) flow diagram. ${ }^{20}$

\section{Data Extraction}


Data will be extracted from selected articles using a data extraction tool within Covidence. ${ }^{19}$ The data extracted will include specific details about the participants, concept, context, study method, and key findings to the review objectives. An outline of the data extraction table is provided below (Table 2). The data extraction tool will be modified and revised as necessary during the process of extracting data from each included evidence source. This will require all reviewers to revisit any previous articles extracted to ensure any additional information in those sources is identified. Any modifications to the data extraction table will be detailed within the scoping review. Any disagreements that arise between the reviewers will be resolved through discussion, or with an additional reviewer. If appropriate, authors of papers will be contacted to request missing or additional data, where required.

Table 2

Data extraction table

\begin{tabular}{|ll|}
\hline General information & Title \\
\cline { 2 - 2 } & Year of publication \\
\hline Author/s \\
\hline Characteristics of included & Journal, Volume, Issue, Pages \\
\hline Country in which the study was conducted \\
\hline Aim/purpose of study \\
\hline Study design \\
\hline Age group \\
\hline Ethnicity \\
\hline Gender \\
\hline Culture \\
\hline Māori or Indigenous \\
\hline Definition of safety strategies \\
\hline $\begin{array}{l}\text { Safety strategy development described: Consumer-based, service- } \\
\text { based or policy-based }\end{array}$ \\
\hline Description of safety strategy evaluation \\
\hline Description of safety strategies during CoviD-19 \\
\hline Safety strategies for Māori identified \\
\hline Safety strategies for Indigenous populations identified \\
\hline Level of response (masks, restrictions, lockdown) \\
\hline
\end{tabular}




\section{Data Analysis and Presentation}

The results will be presented using a Preferred Reporting Items for Systematic Reviews and MetaAnalyses (PRISMA) flow diagram and follow the PRISMA-ScR checklist ${ }^{2}$ for scoping reviews. Data will be analysed using a process of coding ${ }^{21}$ the safety strategies identified within the literature, alongside the perceived effectiveness of these strategies by women and family violence prevention advocates. The potential application of the strategies within an Aotearoa/New Zealand context will also inform data analysis. Findings of this review will be validated with family violence specialists connected with the isafe project. Strategies identified within this scoping review will be published in journals and be incorporated within the isafe online tool.

\section{Pilot}

A pilot was completed with 20 articles selected from searches completed within three databases. Three team members identified three articles to proceed to a full text review. In the event of any ambiguity as to whether to include an article in the full text review, a decision was made to include the article if there is any doubt. Three reviewers completed the full text review, using the Covidence programme ${ }^{19}$ to complete data extraction. Based on this pilot, further search terms were identified. The data extraction tool was modified to ensure additional information related to strategies focussed on supporting Indigenous populations would be identified within the review.

\section{Patient and Public Involvement statement}

Informed by the findings of the scoping review, the research team will develop a plan for stakeholder consultation, including input from the Community Advisory Group. The purposes of the consultation will include sharing preliminary findings and considering the potential application of strategies within Aotearoa.

\section{Strengths And Limitations Of This Study}

- The short timeframe for published peer reviewed articles since the onset of COVID-19 may limit the volume of eligible studies within this review.

- The inclusion of grey literature will support more timely access to practical strategies supporting women experiencing violence during COVID-19.

- The quality of evidence and risk of bias will not be evaluated as part of this scoping review.

- The context surrounding the strategies identified will be examined in relation to the appropriateness for use in New Zealand.

\section{Declarations}

\section{Acknowledgements}


The authors would like to acknowledge the Community Advisory Group for isafe who provide advice and guidance on the project and are supportive of this review. We would also like to acknowledge the support of Yitong Qin who worked with us on a summer student scholarship.

\section{Authors' Contributions}

ZT was the primary authors of the protocol. TBU, SNR and JKM contributed to scoping review design and protocol revisions. ZT, TBU and SNR piloted the study protocol. All authors approved the final protocol manuscript.

\section{Funding}

This work was supported by the Ministry of Business Innovation and Employment grant number CIAF1360 contract/AUTX1907 project.

\section{Conflicts of Interest}

There are no conflicts of interest in this project.

\section{References}

1. Emezue C. Digital or digitally delivered responses to domestic and intimate partner violence during COVID-19. JMIR Public Health Surveillance. 2020;6(3):e19831.

2. Peters MDJ, Marnie C, Tricco AC, et al. Updated methodological guidance for the conduct of scoping reviews. JBI Evidence Synthesis. 2020;18(10):2119-2126.

3. Arksey H, O'Malley L. Scoping studies: towards a methodological framework. International Journal of Social Research Methodology. 2005;8(1):19-32.

4. Levac D, Colquhoun H, O'Brien KK. Scoping studies: advancing the methodology. Implementation Science. 2010;5(1):69.

5. Parker EM, Gielen AC. Intimate partner violence and safety strategy use: Frequency of use and perceived effectiveness. Women's Health Issues. 2014;24(6):584-593.

6. World Health Organisation. Violence against women. https://www.who.int/news-room/factsheets/detail/violence-against-women. Published 2017. Accessed.

7. Campbell JC. Health consequences of intimate partner violence. The Lancet. 2002;359(9314):13311336.

8. Coker AL, Crawford TN, Smith PH, Whitaker DJ, Le B, Flerx VC. Effect of an in-clinic IPV advocate intervention to increase help seeking, reduce violence, and improve well-being. Violence Against Women. 2012;18(1):118-131.

9. Kahui S, Snively S. Measuring the economic costs of child abuse and intimate partner violence to New Zealand. Wellington, N.Z.: MoreMedia Enterprises; 2014. 
10. Fanslow JL, Robinson EM. Sticks, stones, or words? Counting the prevalence of different types of intimate partner violence reported by New Zealand women. Journal of Aggression, Maltreatment \& Trauma. 2011;20(7):741-759.

11. Foster $\mathrm{H}$, Fletcher A. Impact of COVID-19 on women and children experiencing domestic and family violence and frontline domestic and family violence services: summary report. Haymarket: Women's safety NSW;2020.

12. NZ Family Violence Death Review Committee. Fifth data report: January 2009 to December 2015. 2017.

13. Spangaro J, Herring S, Koziol-Mclain J, Rutherford A, Frail M-A, Zwi AB. 'They aren't really black fellas but they are easy to talk to': Factors which influence Australian Aboriginal women's decision to disclose intimate partner violence during pregnancy. Midwifery. 2016;41:79-88.

14. Usher K, Bhullar N, Durkin J, Gyamfi N, Jackson D. Family violence and COVID-19: Increased vulnerability and reduced options for support. International Journal of Mental Health Nursing. 2020;29(4):549-552.

15. Agüero JM. COVID-19 and the rise of intimate partner violence. World Development. 2021;137:105217.

16. Landis D. Gender-based violence and COVID-19: The complexities of responding to "the shadow pandemic". CARE USA;2020.

17. Evans ML, Lindauer M, Farrell ME. A pandemic within a pandemic - Intimate partner violence during Covid-19. New England Journal of Medicine. 2020;383(24):2302-2304.

18. Colquhoun HL, Levac D, O'Brien KK, et al. Scoping reviews: time for clarity in definition, methods, and reporting. Journal of Clinical Epidemiology. 2014;67(12):1291-1294.

19. Covidence systematic review software. [computer program]. Veritas Health Innovation Melbourne, Australia.

20. Tricco AC, Lillie E, Zarin W, et al. PRISMA Extension for Scoping Reviews (PRISMA-ScR): Checklist and Explanation. Annals of Internal Medicine. 2018;169(7):467-+.

21. JBI Manual for Evidence Synthesis. In: Aromataris E, Munn Z, eds.: JBl; 2020: https://synthesismanual.jbi.global.

\section{Supplementary Files}

This is a list of supplementary files associated with this preprint. Click to download.

- PRISMAScRChecklistTipaetal2021.docx 